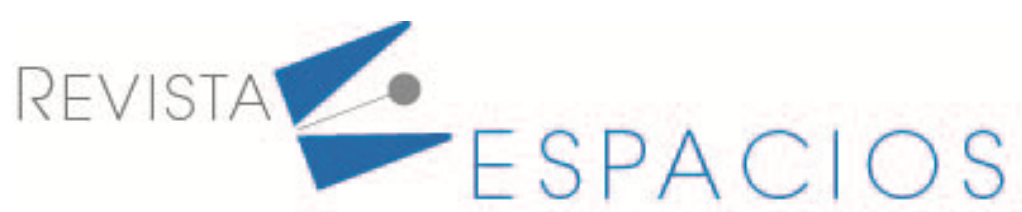

\title{
Percepción de transparencia y corrupción de los ciudadanos colombianos respecto a la administración estatal
}

\section{Perception of transparency and corruption of Colombian citizens regarding the state administration}

\author{
MATALLANA-KUAN, Olga L. ${ }^{1}$ \\ PINEDA-GONZÁLEZ, Myriam L. ${ }^{2}$ \\ BARRETO-BERNAL, Laura M. ${ }^{3}$
}

\begin{abstract}
Resumen
El objetivo es determinar la percepción de transparencia y corrupción que tienen los ciudadanos colombianos respecto a la administración estatal. La metodología es descriptiva, se estudian los índices de Transparencia Colombia, abordando un análisis bibliométrico. Las menores percepciones de transparencia están en divulgación, gestión de programas sociales, gestión de talento humano, políticas anticorrupción, rendición de cuentas y sistema de atención al ciudadano. Concluyendo que el ciudadano empoderado favorece las prácticas de integridad y transparencia.

Palabras clave: transparencia; administración pública; corrupción
\end{abstract}

\begin{abstract}
The objective is to determine the perception of transparency and corruption that Colombian citizens have regarding the state administration. The methodology is descriptive, the Transparency Colombia indices are studied, addressing a bibliometric analysis. The lowest perceptions of transparency are in disclosure, management of social programs, management of human talent, anti-corruption policies, accountability and the citizen service system. Concluding that the empowered citizen favors the practices of integrity and transparency.

Key words: transparency; public administration; corruption
\end{abstract}

\section{Introducción}

El objetivo del artículo es establecer la percepción de transparencia y corrupción que tienen los ciudadanos colombianos respecto a la administración estatal en sus diferentes niveles, aunque en la actualidad la ciudadanía ejerce una mayor revisión sobre lo público, la vigilancia social y la cultura ciudadana de participación no están ampliamente difundidas; al empoderarse, las personas favorecen las prácticas de integralidadinvolucramiento y manejo adecuado de los recursos.

\footnotetext{
${ }^{1}$ Docente Ocasional - Universidad Nacional Abierta y a Distancia - UNAD. Colombia. olga.matallana@unad.edu.co

${ }^{2}$ Docente Ocasional - Universidad Nacional Abierta y a Distancia - UNAD. Colombia. myriam.pineda@unad.edu.co

${ }^{3}$ Docente Facultad de Derecho de la Universidad Antonio Nariño. Colombia. laura.barreto@uan.edu.co
} 
De esta forma, la difusión de la gestión pública, enmarcada en políticas públicas de gobierno abierto, consolida la intervención e información de los habitantes de una región limitando la corrupción. En la investigación se plantea como hipótesis que la consolidación de los mecanismos de visibilidad, institucionalidad y control de la sanción se relacionan de manera directa con una mejor percepción de transparencia.

El sector público en América Latina ( $\mathrm{AL}$ ), y específicamente en Colombia, se ha caracterizado por su escaso nivel de credibilidad y confianza por parte de los ciudadanos en el actuar gubernamental; el despilfarro, la corrupción y las malas prácticas administrativas que rondan las entidades oficiales de la región y se publicitan a diario en los noticieros, contribuyen a que la situación se arraigue aún más.

En general, si la comunidad tiene una opinión desfavorable respecto a las políticas públicas, se siente marginada y mal informada, en relación al accionar de estas entidades, puede llegar a considerar a las administraciones como ineficaces, poco transparentes e incoherentes en torno a sus necesidades más apremiantes. Este escenario ha favorecido la aparición de organizaciones de la sociedad civil que contribuyen a la prevención de los hechos de corrupción estatal. Una de las más reconocidas es la organización Transparencia Internacional (TI).

Transparencia Internacional es un organismo gubernamental, no partidista, sin ánimo de lucro, dedicado a combatir la corrupción a nivel internacional. Fundado en 1993 con el fin de tratar el tema de manera prioritaria en la conciencia global, infundiendo la transparencia y la rendición de cuentas como normas públicamente observadas. Trabaja con la Organización para la Cooperación y el Desarrollo Económicos (OCDE) y la delegación de la Unión Europea, con el fin de fortalecer el marco político y legal en los distintos países (Transparencia Internacional, 2017). TI cree que la sociedad civil debe liderar la lucha global anticorrupción, pues muchas veces los gobiernos y el sector privado forman parte del problema de la corrupción. Para esta organización la corrupción representa "todo abuso de un poder público con fines privados" (Transparencia Internacional 1998: IV), sin embargo, la definición resulta tan amplia que es poco operativa. Visto así el término puede incluir una gran variedad de actos ilícitos, como el soborno, nepotismo, extorsión, fraude, tráfico de influencias, cohecho, favoritismo, lavado de dinero, etcétera.

El presente artículo contempla, en la primera sección, la conceptualización y efectos de la corrupción revisando la posición de autores reconocidos en el tema. En la segunda sección, se aborda literatura científica que ilustra los conceptos de visibilidad, institucionalidad, control y sanción, así como su relación con el fenómeno de estudio. Posteriormente, se presentan los resultados del análisis sobre: (1) la percepción de transparencia en las instituciones colombianas objeto de estudio y (2) la revisión de las líneas temáticas realizada con la herramienta Vosviewer, finalizando con la discusión y las conclusiones.

La literatura científica indica que la causa de la corrupción es la codicia, por tanto, se trata de un fenómeno estructural, es decir, cultural (Arredondo, 2019). Si se analiza su relación con variables económicas y sociales se destacan los estudios Rose-Ackerman (2001), quien sostiene que la elevada corrupción limita la inversión y el desarrollo y conduce a un gobierno ineficaz con marcadas desigualdades económicas. Respecto a las consecuencias de problema Rothstein (2003), afirma que los niveles altos de corrupción y el bajo índice de calidad de la administración pública que se encuentran en la mayoría de los países contemporáneos resulta tener efectos devastadores en la prosperidad, el bienestar social, la salud, la satisfacción con la vida y, por supuesto, en la confianza de los ciudadanos, de esta manera son innegables los efectos transversales de este fenómeno y su repercusión en los niveles de bienestar de los ciudadanos y las regiones. 
Philp (2001) indica dos caminos para el control de la corrupción, el primero e se centra en el escrutinio y la supervisión de las funciones gubernamentales y el segundo analiza la integridad profesional de quienes ejercen autoridad.

En Colombia uno de los estudios más completos e integrales sobre la corrupción titulado "marco conceptual, diagnóstico y propuestas de política" financiado por Fedesarrollo, da cuenta de las principales formas de corrupción en el país, tales como el pago por el acceso a ciertos beneficios (contratos, subsidios etc.), y el pago para evadir costos (sanciones, impuestos etc.), el mismo estudio identifica la relación entre los efectos las prácticas corruptas con el el subdesarrollo de los países en donde no se puede consolidar el estado de derecho y se generan altos costos morales para la sociedad (Newman y Ángel, 2017).

\subsection{Visibilidad, institucionalidad y control y sanción y su relación con la percepción de transparencia}

Una de las mayores controversias al estudiar la percepción de transparencia es que por tratarse de un fenómeno subjetivo, no se puede medir de manera apropiada, no existen estadísticas que permitan medir adecuadamente estos fenómenos y si bien los estudios empíricos pueden mostrar relaciones causales, no es posible extrapolar la información para analizar casos a nivel general; sin embargo, hay acuerdos respecto a la importancia de los datos abiertos para la comunicación del quehacer gubernamental.

Al estudiar la transparencia, se destacan las investigaciones de Kosack y Fung (2014), quienes indican que esta concepción se orienta a la libertad o derecho de solicitar información que tienen los ciudadanos y la obligación de los gobiernos a proporcionarla, potencializando así el espíritu democrático. La divulgación y visibilización de información empodera a las comunidades y responsabiliza a los funcionarios públicos, reduciendo la mala gestión de los recursos y conduciendo, eventualmente, a una gobernanza responsable, receptiva y eficaz. El artículo "Buena administración, transparencia y eficiencia: evidencia de los municipios de Colombia" resalta la dificultad de abordar conceptos abstractos para medirlos de forma cuantitativa y enfatiza en la relación de la transparencia con el cumplimiento de la normatividad y la eficiencia administrativa (Ortiz y Ordoñez, 2019).

Cunill (2010), advierte la correspondencia entre la institucionalidad y la determinación de políticas públicas orientadas a garantizar los derechos de los ciudadanos, ya que son las instituciones las que expiden las reglamentaciones para penalizar o estimular el comportamiento social, postula que "hay tres atributos insoslayables que debe reunir la institucionalidad pública: accountability (o responsabilización), gobernanza sistémica y espacio público" (pág. 5). Con esta misma orientación, Cerrillo ( 2012) sostiene que la lucha contra la corrupción exige impulsar decididamente un cambio de cultura institucional, que favorezca un sistema de controles y equilibrios basados en una mayor información de las administraciones gubernamentales a sus comunidades.

Respecto a control y sanción, Cunill (2003) menciona que "la administración recibe constantes demandas para ampliar la transparencia de su operación y para incorporar a la población de distintos modos en la formulación, seguimiento y evaluación de sus acciones" (pag.7). En este sentido considera que la responsabilidad y el control van de la mano, el control implica revisar cómo es ejercido el poder pero la responsabilidad supone una relación entre resultados y sanciones (directas) e involucra controlar ex-ante y ex-post el actuar estatal. En términos de control, la denuncia y las acciones ciudadanas de interés público pueden ser las herramientas para exigir los derechos ciudadanos a los gobiernos estatales. 


\section{Metodología}

La presente investigación se orienta hacia un tipo de estudio descriptivo, ya que reseña rasgos, cualidades o atributos del objeto de estudio, que son las entidades públicas, al respecto vale la pena citar a Pliscoff y Monje (2003), quienes afirman que la investigación sobre gestión pública mezcla argumentos de dos tipos: descriptivos y propositivos, ya que está orientada a combinar la descripción con la acción, al tratar de reconocer la forma en que se lleva una organización y del mismo modo, delimita formas y lineamientos de cómo deben guiarse.

En lo que refiere al método de investigación en gestión pública, Pliscoff, y Monje (2003), plantean la investigación comparada como pertinente, porque permite analizar el objeto o fenómeno específico y la comparación de fenómenos sociales a distintos niveles de la estructura social, buscando explicar las diferencias y semejanzas entre países, explorando patrones, procesos y regularidades existentes. El estudio se basa en el análisis de los datos del Índice de Transparencia nacional y de los organismos públicos del orden ejecutivo, legislativo y judicial de Colombia, se analizan las cifras de los Departamentos, Municipios, Contralorías departamentales, Organismos de Control, Ministerios y entidades nacionales.

La técnica de investigación es cuantitativa, al hacer uso de bases de datos libres emanadas por Transparencia Colombia (2017), para la vigencia de 2015-2016. El Índice de Transparencia de las Entidades Públicas cuenta con tres instrumentos de evaluación: 1. El Índice de Transparencia Nacional que evalúa entidades de las tres ramas del poder público, los órganos de control y los Ministerios; 2. El Índice de Transparencia Departamental, el cual evalúa la gestión centralizada de las gobernaciones y las contralorías departamentales y 3 . El Índice de Transparencia Municipal, diseñado para evaluar la gestión central de las alcaldías. (Transparencia Colombia, 2017)

En los datos estudiados, el peso de cada factor es visibilidad $30 \%$, institucionalidad $40 \%$ y control y sanción 30 \%. Los niveles de los riesgos de corrupción se evalúan de 1 a 100, donde los niveles más altos tienen menores riesgos de corrupción: el nivel muy alto va de 0 a 44,4, el nivel alto va de 44,5 a 59,9, el nivel medio va de 60 a 74,4 , el nivel moderado va de 74,5 a 89,4, y el nivel bajo va de 89,5 a 100. (Índice de Transparencia Municipal, 2017, pág. 6). La Corporación Transparencia por Colombia, evaluó 75 entidades del orden nacional, 28 municipios, 32 contralorías y 32 Gobernaciones, en total 167 entidades. A partir de esta información, se construyó una base de datos por nivel territorial discriminando como variable endógena la percepción de transparencia y variables exógenas: visibilidad, institucionalidad y control y sanción, evaluándolas de acuerdo al comportamiento del promedio porcentual de 16 subindicadores. (Tabla 1).

Los datos de análisis se toman del promedio de cada factor y entidad acorde a los datos de Transparencia Colombia, así se promedian los indicadores y subindicadores de 28 alcaldías, 32 gobernaciones, 32 contralorías y 75 entidades del orden nacional. (Tabla 3 y 4 ). 
Tabla 1

Variables y subindicadores de estudio

\begin{tabular}{|c|c|c|}
\hline Variable & Descripción & Subindicadores \\
\hline Visibilidad & $\begin{array}{l}\text { Es la capacidad de una entidad para } \\
\text { hacer públicas sus políticas, } \\
\text { procedimientos y decisiones, de } \\
\text { manera suficiente, oportuna, clara y } \\
\text { adecuada. }\end{array}$ & $\begin{array}{l}\text { Divulgación de información pública } \\
\text { Divulgación de la gestión administrativa } \\
\text { Divulgación de la gestión presupuestal y financiera } \\
\text { Divulgación de trámites y servicio al ciudadano } \\
\text { Divulgación de programas sociales }\end{array}$ \\
\hline Institucionalidad & $\begin{array}{l}\text { Es la capacidad de una entidad para } \\
\text { lograr que los servidores públicos y la } \\
\text { administración en su conjunto } \\
\text { cumplan con normas y estándares } \\
\text { establecidos para los procesos de } \\
\text { gestión. }\end{array}$ & $\begin{array}{l}\text { Políticas, medidas y estrategias anticorrupción } \\
\text { Comportamiento ético } \\
\text { Gestión de la planeación } \\
\text { Gestión de la contratación } \\
\text { Gestión del Talento Humano } \\
\text { Gestión de Programas sociales }\end{array}$ \\
\hline Control y sanción & $\begin{array}{l}\text { Es la capacidad para generar } \\
\text { acciones de control y sanción } \\
\text { mediante procesos internos, por } \\
\text { acción de los órganos de control y } \\
\text { espacios de participación ciudadana. }\end{array}$ & $\begin{array}{l}\text { Sistema de atención al ciudadano } \\
\text { Rendición de cuentas a la ciudadanía } \\
\text { Participación y control social } \\
\text { Control institucional } \\
\text { Control Interno de Gestión y Disciplinario }\end{array}$ \\
\hline
\end{tabular}

Fuente: Elaboración propia basada en datos de Transparencia Colombia.

\subsection{Métodos y herramientas bibliométricas}

Con la intención de fortalecer el análisis y la discusión de resultados, se plantea la revisión sistemática de literatura a partir de técnicas bibliométricas, empleando ecuaciones de búsqueda con las palabras clave determinadas en las variables estudiadas para conocer la producción académica en el tema, tomando como fuente la plataforma Scopus, y usando mapas de visualización de ciencia para establecer y analizar redes de coocurrencia y comprender con mayor facilidad el concepto y las tendencias científicas. Al respecto Martínez-Toro, Roco Bautista, Romero -Riaño, Galeano Barrera, Guerrero y Parra - Valencia (2018), explican que "la coocurrencia de palabras clave indica la relación de proximidad entre dos o más palabras clave dentro de un documento, se representa, mediante las gráficas de redes de enlace entre términos" (Pág. 366).

En la selección de unidades de análisis, se plantea un protocolo de revisión con criterios de inclusión y exclusión, se incluyen artículos publicados a partir de 2016 a 2020, cuyo título o resumen se relacione con el objeto de investigación y sean artículos o revisiones, se excluyen todas las publicaciones que no correspondan a las áreas temáticas estudiadas. Para el establecimiento de la ecuación de búsqueda se emplearon palabras claves acordes a las categorías establecidas en la Tabla 2 y operadores booleanos AND, OR, NOT para realizar las ecuaciones y depurar los registros.

Para que la muestra fuera representativa de los grupos temáticos, se seleccionaron palabras en inglés utilizando la opción KW con una frecuencia mayor o igual a 3 veces. Cómo Herramienta tecnológica de soporte se empleó el programa VOSviewer que permite identificar y visualizar la estructura intelectual, las relaciones y dinámica de la investigación en el tema. Siguiendo la metodología descrita se revisaron los resúmenes para establecer las unidades de análisis pertinentes dando sentido al contenido buscado en los artículos y expresándolo en un mapa gráfico que muestra los vínculos de los términos o temas más comunes y sus años de publicación. 
Tabla 2

Palabras clave

\begin{tabular}{lll}
\hline AND & & \\
\hline & "public administration" & Visibility \\
& "public administration" & institutional OR institutionality \\
OR $\quad$ "public administration" & "control " \\
\hline
\end{tabular}

Como limitante del estudio se plantea que medir el fenómeno de la corrupción y la percepción de transparencia es complejo, ya que la información emanada por transparencia internacional se basa en encuestas que pueden tener cierto grado de subjetividad. Respecto al análisis bibliométrico la restricción está en analizar una única base de datos, lo que implica una perspectiva específica en la exploración realizada.

\section{Resultados y discusión}

Las entidades territoriales departamentales y municipales del país se caracterizan por su heterogeneidad, cuentan con diversos recursos financieros, humanos e institucionales; diferentes condiciones culturales, económicas y sociales que permiten un mayor o menor desarrollo, son evidentes las brechas en la gestión que impiden una mejor relación entre el gobierno y los ciudadanos. En la Tabla 3 se establecen las entidades públicas mejor y peor valoradas acorde al IT (2015-2016).

En general, las gobernaciones con mejor desempeño son la de Antioquia, Meta y Santander, que presentan un nivel moderado de riesgo de corrupción. Las gobernaciones con mayor riesgo de corrupción fueron las de Choco, Guajira y Guainía. En todos los casos el factor mejor valorado fue el de visibilidad mediante la difusión en redes sociales, páginas web, que potencializan los mecanismos de comunicación externa. La variable más débil es el de institucionalidad, siendo evidente el reto que impone la gestión de lo público y la importancia de la integración con el gobierno central.

En lo que respecta a las alcaldías, las de menor riesgo de corrupción fueron las de Pasto, Pereira y Barranquilla, siendo control y sanción, el factor mejor valorado. Las alcaldías con mayor riesgo de corrupción fueron Puerto Carreño, Santa Marta y Mitú. En este sentido, cabe anotar que la participación ciudadana y el interés de la ciudadanía por lo gubernamental, permiten que se den mayores acciones de inspección y sentido de pertenencia por su municipio encaminando el quehacer público al logro de eficiencia y eficacia en el manejo de los recursos públicos.

Las contralorías con mejores puntuaciones fueron las de Huila, Cundinamarca y Risaralda, los dos primeros presentan elevados indicadores de visibilidad, Risaralda tiene su mejor puntuación en control y Sanción. Las contralorías de mayor riesgo de corrupción fueron Amazonas, Guainía y Vichada, todas con el menor índice en el factor de visibilidad. En entidades del orden nacional las más destacadas fueron la Superintendencia de Sociedades, la Superintendencia Financiera de Colombia y el Departamento Nacional de Planeación - DNP, destacándose en las dos últimas el factor de visibilidad como el más importante, las entidades de mayor riesgo de corrupción fueron la Superintendencia de la Economía Solidaria, la Unidad de Información y Análisis Financiero - UIAF, y Fiscalía General de la Nación siendo bastante bajo el indicador de control y sanción en las 2 últimas.

En conjunto cabe destacar que ninguna de las 167 entidades analizadas obtuvo un nivel bajo en el índice de transparencia. Una vez más ante la corrupción la mejor herramienta es el control social, aunque en términos de visibilidad, de acuerdo a la Corporación Transparencia por Colombia, los procesos más débiles en estas 
instituciones del estado colombianas son los de contratación pública que no cuentan con la debida publicidad y la garantía de tener varios proponentes. El empleo público es bastante débil en los municipios y gobernaciones, en parte porque es el ítem más orientado a la percepción de clientelismo, adicionalmente hay muchos contratistas en las plantas de personal, generando así una brecha salarial con otros empleados. Los indicadores de participación ciudadana y atención al ciudadano son muy frágiles a nivel territorial.

Tabla 3

Índice de transparencia de las entidades públicas colombianas, 2015-2016

\begin{tabular}{|c|c|c|c|c|c|}
\hline Nombre de la Entidad & ITM 2015-2016 & $\begin{array}{l}\text { Nivel de } \\
\text { riesgo }\end{array}$ & $\begin{array}{l}\text { Visibilidad } \\
\text { sobre } 100 \\
\end{array}$ & $\begin{array}{l}\text { Institucionalidad } \\
\text { sobre } 100\end{array}$ & $\begin{array}{c}\text { Control y Sanción } \\
\text { sobre } 100\end{array}$ \\
\hline Gobernación de Antioquia & 85,6 & Moderado & 91,3 & 27,4 & 85,9 \\
\hline Gobernación del Meta & 80,1 & Moderado & 80,1 & 24,0 & 77,1 \\
\hline Gobernación de Santander & 79,2 & Moderado & 86,3 & 25,9 & 72,9 \\
\hline Gobernación de Guainía & 43,0 & Muy Alto & 46,7 & 14,0 & 40,7 \\
\hline Gobernación de La Guajira & 41,7 & Muy Alto & 66,4 & 19,9 & 32,3 \\
\hline Gobernación de Chocó & 30,2 & Muy Alto & 40,1 & 12,0 & 18,1 \\
\hline Alcaldía de Pasto & 77,9 & Moderado & 69,9 & 75,3 & 89,4 \\
\hline Alcaldía de Pereira & 75,5 & Moderado & 67,9 & 78,1 & 79,7 \\
\hline $\begin{array}{l}\text { Alcaldía Distrital de } \\
\text { Barranquilla }\end{array}$ & 74,9 & Moderado & 72,0 & 75,2 & 77,3 \\
\hline Alcaldía de Mitú & 39,7 & Muy alto & 36,7 & 42,0 & 39,7 \\
\hline $\begin{array}{l}\text { Alcaldía Distrital de Santa } \\
\text { Marta }\end{array}$ & 37,7 & Muy alto & 33,3 & 36,6 & 43,8 \\
\hline Alcaldía de Puerto Carreño & 35,4 & Muy alto & 41,6 & 29,3 & 37,3 \\
\hline Contraloría del Huila & 87,1 & Moderado & 99,0 & 75,9 & 90,0 \\
\hline Contraloría de Cundinamarca & 87,0 & Moderado & 94,2 & 80,2 & 89,0 \\
\hline Contraloría de Risaralda & 80,1 & Moderado & 80,7 & 76,1 & 85,0 \\
\hline Contraloría de Vichada & 43,4 & Muy Alto & 18,5 & 57,3 & 49,9 \\
\hline Contraloría de Guainía & 40,6 & Muy Alto & 37,0 & 45,4 & 37,8 \\
\hline Contraloría de Amazonas & 34,6 & Muy Alto & 20,7 & 41,8 & 38,8 \\
\hline $\begin{array}{l}\text { Superintendencia de } \\
\text { Sociedades }\end{array}$ & 86,68 & Moderado & 83,4 & 87,7 & 88,7 \\
\hline $\begin{array}{l}\text { Superintendencia Financiera } \\
\text { de Colombia }\end{array}$ & 84,96 & Moderado & 92,5 & 76,5 & 88,6 \\
\hline $\begin{array}{l}\text { Departamento Nacional de } \\
\text { Planeación - DNP }\end{array}$ & 80,44 & Moderado & 87,7 & 72,6 & 83,7 \\
\hline $\begin{array}{l}\text { Superintendencia de la } \\
\text { Economía Solidaria }\end{array}$ & 52,77 & Alto & 68,8 & 48,4 & 42,5 \\
\hline Fiscalía General de la Nación & 50,93 & Alto & 53,2 & 57,7 & 39,5 \\
\hline $\begin{array}{l}\text { Unidad de Información y } \\
\text { Análisis Financiero - UIAF }\end{array}$ & 47,53 & Alto & 72,9 & 32,4 & 42,3 \\
\hline
\end{tabular}

Fuente: Autor datos de Transparencia Colombia (2017). 


\subsection{Resultados por factor}

El primer factor a discutir, es la visibilidad, en la Tabla 4, se indica el comportamiento de esta variable con sus sub variables explicativas. En términos de visibilidad las entidades nacionales tienen una mejor ponderación (77.2\%), las contralorías son las clasificadas como menos visibles (58.9\%), En los datos de visibilidad, la sub variable de divulgación y gestión presupuestal y financiera es la mejor ponderada en alcaldías, gobernaciones y entidades nacionales. Las contralorías por su parte tienen mejor ponderación en la divulgación de la gestión administrativa. En las alcaldías el factor menos valorado fue la divulgación de programas sociales, en las gobernaciones, entidades nacionales y contralorías, el factor menos valorado fue la divulgación de información pública (Tabla 4).

Tabla 4

Promedio de factores de Visibilidad, institucionalidad y Control y sanción

\begin{tabular}{|c|c|c|c|c|c|c|c|}
\hline \multicolumn{8}{|c|}{ Factor Visibilidad } \\
\hline Entidades & Visibilidad \% & $\begin{array}{l}\text { Divulgación de } \\
\text { información } \\
\text { pública }\end{array}$ & $\begin{array}{l}\text { Divulgación de la } \\
\text { gestión } \\
\text { administrativa }\end{array}$ & $\begin{array}{l}\text { Divulgación de } \\
\text { la gestión } \\
\text { presupuestal y } \\
\text { financiera }\end{array}$ & $\begin{array}{l}\text { Divulgación } \\
\text { de trámites y } \\
\text { servicio al } \\
\text { ciudadano }\end{array}$ & $\begin{array}{c}\text { Divulgación } \\
\text { de } \\
\text { programas } \\
\text { sociales }\end{array}$ & \\
\hline Alcaldías & 59,3 & 52,8 & 63,9 & 69,0 & 68,0 & 28,6 & \\
\hline Gobernaciones & 70,7 & 48,5 & 70,2 & 79,8 & 75,8 & $\mathrm{~N} / \mathrm{A}$ & \\
\hline $\begin{array}{l}\text { Entidades } \\
\text { Nacionales }\end{array}$ & 77,2 & 60,4 & 74,1 & 86,4 & 81,9 & $\mathrm{~N} / \mathrm{A}$ & \\
\hline Contralorías & 58,9 & 37,4 & 68,3 & 60,4 & 58,9 & $\mathrm{~N} / \mathrm{A}$ & \\
\hline \multicolumn{8}{|c|}{ Factor institucionalidad } \\
\hline Entidades & $\begin{array}{c}\text { Institucionalidad } \\
\%\end{array}$ & $\begin{array}{c}\text { Políticas, } \\
\text { medidas y } \\
\text { estrategias } \\
\text { anticorrupción }\end{array}$ & $\begin{array}{c}\text { Comportamiento } \\
\text { ético }\end{array}$ & $\begin{array}{c}\text { Gestión de la } \\
\text { planeación }\end{array}$ & $\begin{array}{l}\text { Gestión de la } \\
\text { contratación }\end{array}$ & $\begin{array}{c}\text { Gestión del } \\
\text { Talento } \\
\text { Humano }\end{array}$ & $\begin{array}{c}\text { Gestión de } \\
\text { Programas } \\
\text { sociales - } \\
\text { Gestión de } \\
\text { control fiscal } \\
\text { en contralorías }\end{array}$ \\
\hline Alcaldías & 54,6 & 52,7 & 55,9 & 65,2 & 62,4 & 48,0 & 42,5 \\
\hline Gobernaciones & 57,2 & 53,4 & 53,9 & 66,0 & 60,9 & 52,6 & N/A \\
\hline $\begin{array}{l}\text { Entidades } \\
\text { Nacionales }\end{array}$ & 64,2 & 51,3 & 51,7 & 54,9 & 73,1 & 72,1 & N/A \\
\hline Contralorías & 63,6 & 50,6 & 60,6 & 59,9 & 77,1 & 56,1 & 71,7 \\
\hline \multicolumn{8}{|c|}{ Factor control y sanción } \\
\hline Entidades & $\begin{array}{l}\text { Control y } \\
\text { Sanción \% }\end{array}$ & $\begin{array}{l}\text { Sistema de } \\
\text { atención al } \\
\text { ciudadano - } \\
\text { Sistema de PQR } \\
\text { en entidades } \\
\text { nacionales }\end{array}$ & $\begin{array}{l}\text { Rendición de } \\
\text { cuentas a la } \\
\text { ciudadanía }\end{array}$ & $\begin{array}{c}\text { Participación y } \\
\text { control social }\end{array}$ & $\begin{array}{c}\text { Control } \\
\text { institucional }\end{array}$ & $\begin{array}{c}\text { Control } \\
\text { Interno de } \\
\text { Gestión y } \\
\text { Disciplinario }\end{array}$ & \\
\hline Alcaldías & 57,3 & 48,0 & 46,8 & 63,4 & 52,4 & 71,7 & \\
\hline Gobernaciones & 58,0 & 37,9 & 47,7 & 69,0 & 57,0 & 73,3 & \\
\hline $\begin{array}{l}\text { Entidades } \\
\text { Nacionales }\end{array}$ & 64,5 & 67,7 & 54,1 & 58,4 & $\mathrm{~N} / \mathrm{A}$ & 85,0 & \\
\hline Contralorías & 60,9 & 57,3 & 44,3 & 70,1 & $\mathrm{~N} / \mathrm{A}$ & 75,8 & \\
\hline
\end{tabular}

Al revisar el factor de institucionalidad las entidades nacionales cuentan con la mejor ponderación (64.2\%), la más baja corresponde a las alcaldías (54.6\%). La gestión de la planeación se percibe como el mejor indicador en alcaldías y gobernaciones, en las entidades nacionales y contraloría es la gestión de la contratación la que tiene un mejor indicador. En las alcaldías la gestión de programas sociales se percibe como el factor más débil, en las gobernaciones es la gestión del talento humano y en las instituciones nacionales y contraloría son las políticas anticorrupción las percibidas con menor puntuación (Tabla 4). 
En factor control y sanción, las entidades nacionales cuentan con la mejor percepción (64.5\%) y la más baja corresponde a las alcaldías (57.3\%). En la sub variable de control interno y disciplinario se percibe como la mejor valorada en alcaldías, gobernaciones, entidades nacionales y contralorías. La rendición de cuentas a la ciudadanía presentó la percepción más baja en alcaldías, entidades nacionales y contralorías. En el caso de las gobernaciones el factor menor valorado fue el sistema de atención al ciudadano (Tabla 4).

\subsection{Análisis Bibliométrico}

\subsubsection{Análisis de co-ocurrencia Visibilidad}

En el estudio bibliométrico del tema se identificaron un total de 21 documentos publicados, 10 ítems y 3 clúster temáticos relacionados con "public administration" and visibility; como se muestra en la Figura 1. Se puede indicar que las líneas de investigación se han orientado a temas como información y comunicación y tecnologías de la información. Al revisar los resúmenes de las publicaciones se establece que la visibilidad de la información estatal permite modernizar el estado y controlar la gestión, facilitando la transparencia y la posibilidad de transferir las prácticas a nuevos escenarios. Se indica que mejores entornos digitales y condiciones de desarrollo informativo permiten a los ciudadanos involucrarse. La difusión del quehacer de las administraciones públicas mediante la tecnología genera mayor conciencia y posibilita espacios de planificación abierta y participación de la comunidad en la toma de decisiones.

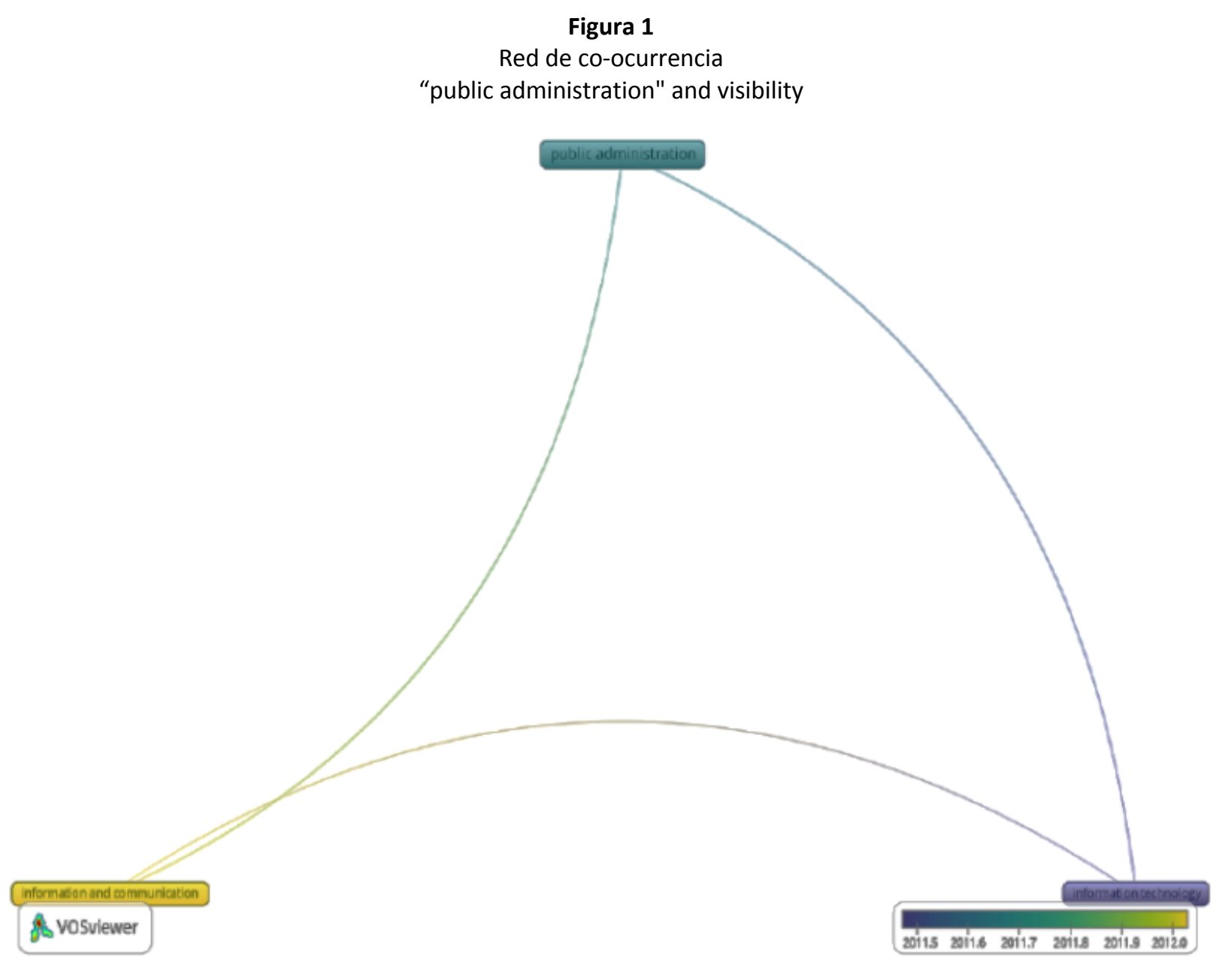

Fuente: Elaboración propia Vos Viewer 


\subsubsection{Análisis de co-ocurrencia Institucionalidad}

Con la búsqueda "public administration" and institutional OR institutionality se trabajaron 674 unidades de análisis, 82 términos clasificados en 8 clúster temáticos (Figura 2). Se aprecia que la institucionalidad es percibida de diferente manera según el contexto que se analice ya que la efectividad de las instituciones públicas varía de acuerdo al país o región; sin embargo, es posible identificar la relación del término con la participación de la sociedad civil y los procesos democráticos. También puede apreciarse en los artículos analizados que especialmente los gobiernos regionales ven en las actividades de rendición de cuentas un mecanismo para soportar la institucionalidad. Hay una relación fuerte en el estudio de la institucionalidad y las teorías de administración pública, cómo han variado a lo largo de los años, hasta convertirse en un híbrido de diferentes corrientes. En América Latina, la administración pública ha pasado por transformaciones ideológicas importantes, las reformas respecto a la modernización del estado han atravesado de la administración y cultura burocrática hacia una cultura gerencial orientada por los principios de la nueva gestión pública (NGP) (Prats, 1998). Actualmente dado el acceso masivo a los datos se vienen posicionando nuevos paradigmas, como el de Gobierno abierto que se enfoca a fortalecer el vínculo entre gobernantes, administración y sociedad mediante una mayor participación ciudadana (Dassen, Vieyra, Molina, Ramírez, Díaz, Elena, \& De Michele (2012). En el análisis bibliométrico también se resaltan términos relacionados como el enfoque de gobernanza, la rendición de cuentas y el gasto público.

\section{Figura 2}

Grafico de red de co-ocurrencia

"public administration" and institutional OR institutionality

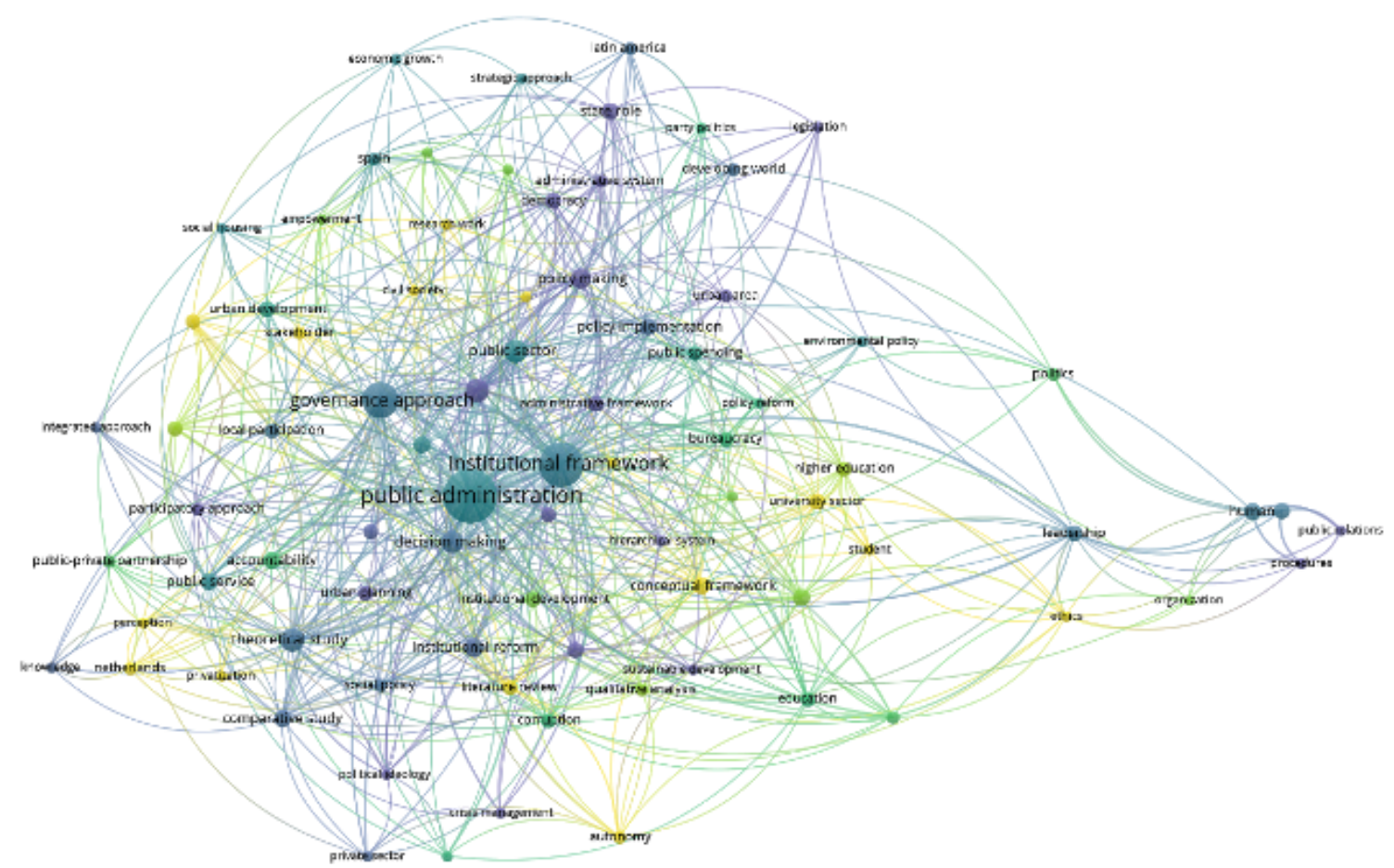

A vosviewer

Fuente: Elaboración propia Vos Viewer 
Figura 3

Gráfico de red de co-ocurrencia "public administration" and institutional OR institutionality-Nodos tematicos

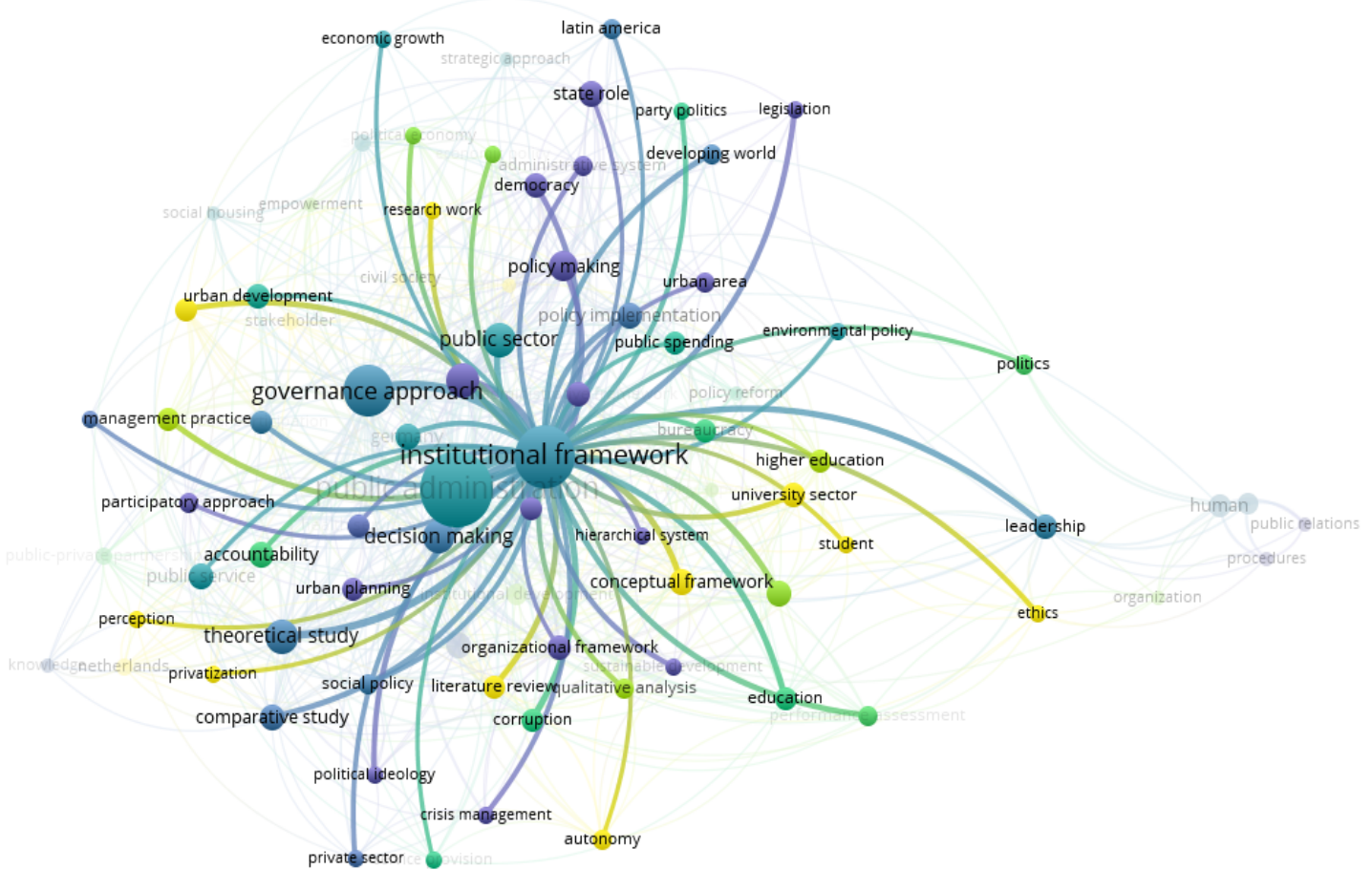

Fuente: Elaboración propia Vos Viewer

\subsubsection{Análisis de co-ocurrencia Control y Sanción}

Con la búsqueda "public administration" AND"control " se revisaron 498 documentos, se clasificaron 87 términos en 6 clúster temáticos (Figura 3). Los mecanismos de control tienen estrecha relación con la confianza institucional de los ciudadanos; sin embargo, en ocasiones no permiten determinar con claridad al culpable de una mala calidad del servicio público, tampoco se establece de manera efectiva cómo se puede mejorar el desempeño, ni se establecen esquemas de perfeccionamiento para lograr mayor eficiencia y legitimidad, aunque hoy por hoy se cuenta con comunidades activas, conscientes de que los bienes públicos son de todos, que manifiestan sus inconformidades en redes sociales y en protestas sociales, aún se requiere fortalecer modelos de control que redunden en acciones de mejora para limitar los abusos de poder y corrupción, fortaleciendo la democracia y eficacia en el quehacer de las administraciones públicas 
Figura 4

Gráfico de red de co-ocurrencia

"public administration" and control

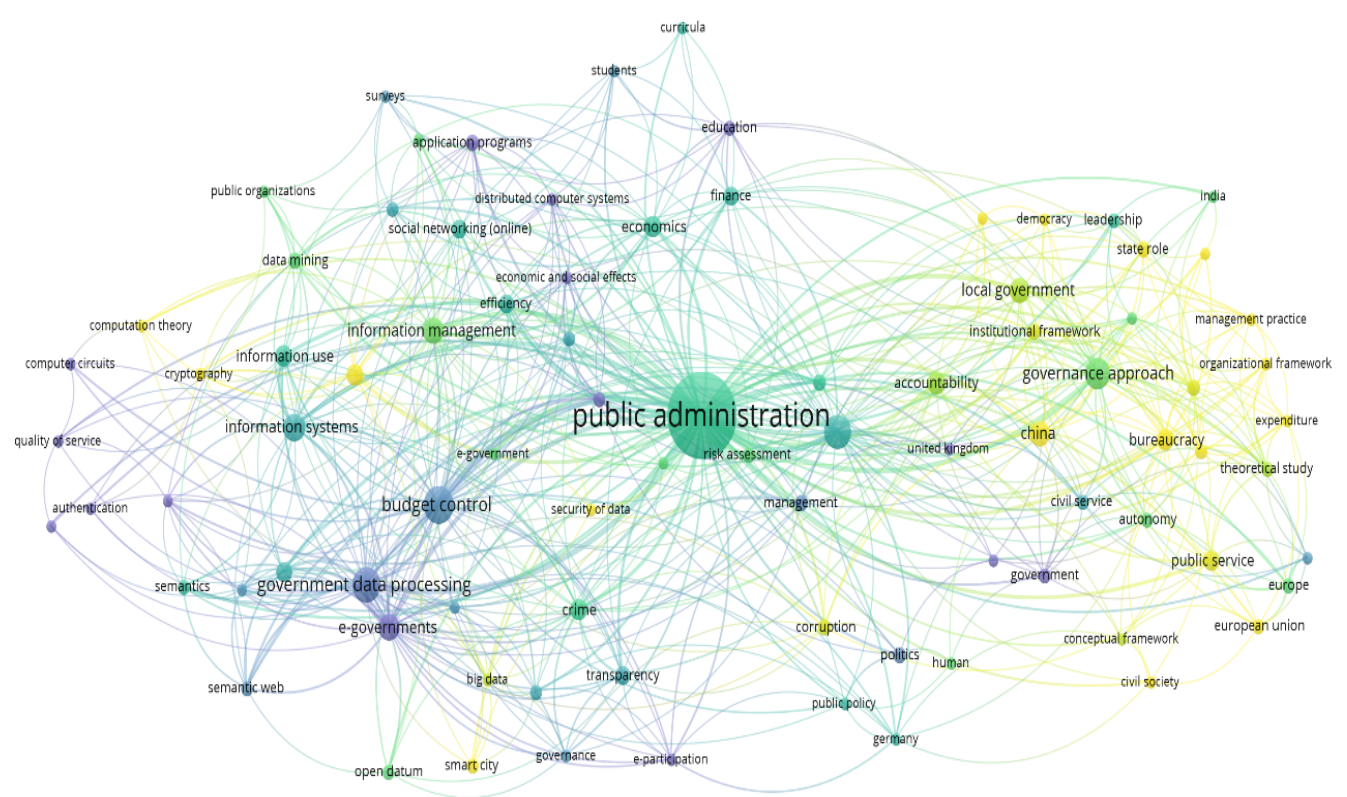

\& Vosviewer

Fuente Elaboración propia, Vos viewer

Figura 5

Gráfico de red de co-ocurrencia

"public administration" and control-Nodos Temáticos
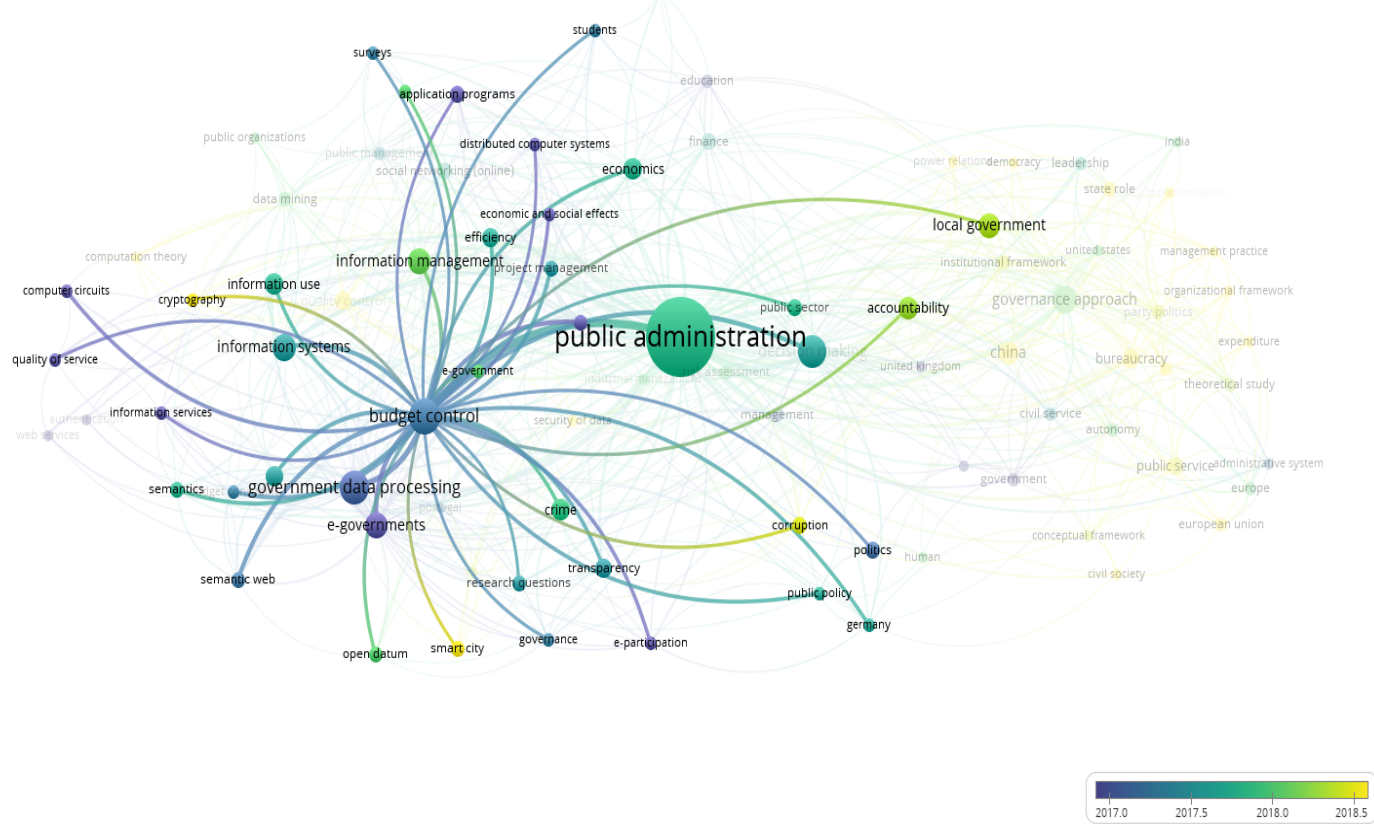

Fuente Elaboración propia, Vos viewer 
No es fácil determinar empíricamente la relación de los mecanismos de visibilidad, institucionalidad y control y sanción con la percepción de transparencia, pues la percepción de transparencia envuelve la posibilidad de hacer visible la gestión de las entidades públicas y también implica que los ciudadanos participen y conozcan sus derechos y deberes. La transparencia se materializa a partir del acceso público a datos o información, esto es, al considerar la existencia o no de información y de sistemas que la generan, indicando lo que son, hacen, utilizan y producen las dependencias de gobierno o, incluso, sobre actos o productos de actores privados Fox (2007). Esta debe arrojar luz sobre las normas, planes, procesos y acciones, asegurando que los funcionarios públicos, gerentes, miembros del directorio y los empresarios actúan de forma visible y comprensible e informan sobre sus actividades, lo que implica que la sociedad pueda pedirles cuentas (Transparencia Internacional, 2017). En este sentido en el país se está avanzando en acciones de transparencia activa donde la entidad de manera voluntaria informa a la ciudadanía acerca de sus procesos y sus ejecutorias (Católico \& Santos, 2018).

En los resultados del estudio, la percepción más alta de visibilidad se dio en la divulgación de información financiera y presupuestal, pues la normativa vigente obliga a las entidades a hacer públicas sus actuaciones y el fomento de las tecnologías de información fortalece las posibilidades de que los ciudadanos del común conozcan de primera mano cómo se realiza la gestión financiera en las entidades territoriales, sin embargo y como se determinó en el análisis bibliométrico informar no implica que se estén tomando las mejores determinaciones. El factor más débil identificado en términos de visibilidad, fue en contraste la divulgación de la información pública, no existen en las entidades esquemas fortalecidos para divulgar la información, ni existe periodicidad en las informaciones que se publican, aun muchos documentos públicos que siguen manteniendo el carácter reservados o clasificados y no son de acceso a los ciudadanos, de otra parte los procesos de gestión documental aunque se han fortalecido requieren ser más fuertes y en muchos casos digitalizarse.

El concepto de visibilidad va de la mano con la idea de Gobierno Abierto orientada a reinventar y reorganizar el sistema hacia la apertura a la ciudadanía. El gobierno abierto es una estrategia de cultura de la comunicación, donde el estado pone la información a disposición de la sociedad y comunica su actuar de manera transparente, a este respecto Naser y Ramírez- (2013) aclaran:

Cuando se habla de gobierno abierto, se hace referencia al menos a los siguientes dos ejes: a) promover una cultura de transparencia e institucionalización de acciones que faciliten el acceso a información pública y su potencial reutilización (con fines de control social o político; de generar valor público, cívico o económico, etc.), al mismo tiempo que se facilitan los espacios de fortalecer la integridad pública y la rendición de cuentas; y b) Fortalecer los espacios de participación ciudadana en los asuntos públicos y en la toma de decisiones que les atañen, promoviendo además la colaboración en la búsqueda e implementación de soluciones en un esquema de mayor responsabilidad compartida que pueda aprovechar las capacidades distribuidas y la inteligencia colectiva de los actores sociales (Pág. 13).

Así, el gobierno abierto descansa sobre tres pilares: la transparencia, la participación y La colaboración (Figura 6). Las estrategias de gobierno abierto se orientan en dos ejes: El primero es la apertura de datos públicos (open data), que promueve la innovación y el uso de los datos para nuevas aplicaciones y servicios permitiendo que otras entidades y/o usuarios puedan generar nuevas actividades económicas y agregar valor público, incrementando así la transparencia, la rendición de cuentas y el escrutinio público permanente. El segundo es la apertura de procesos (open process) y el uso de redes sociales (también llamado Gobierno 2.0), cuyo objeto es facilitar la comunicación y la experiencia de los ciudadanos para ayudar en el diseño de políticas y la provisión de servicios públicos y la colaboración (en red) dentro y entre las administraciones públicas, en un entorno de decisiones abiertas (participación) y servicios abiertos (colaboración) (Dassen,\& otros, 2012). 
Figura 6

Pilares del Gobierno abierto

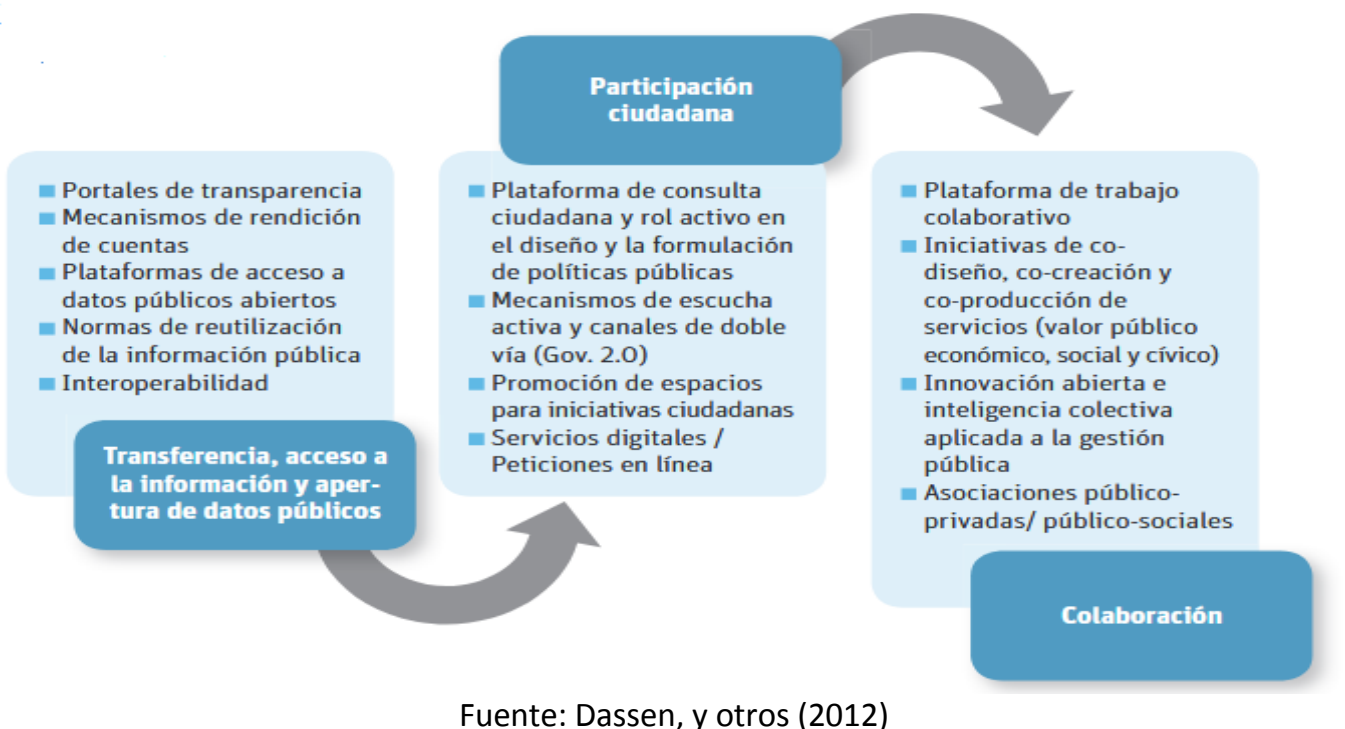

La idea de la transparencia publica, se popularizo en Estados Unidos, concretamente en la Ley de Libertad de Información (FOIA, por sus siglas en inglés), pero fue Barack Obama, durante su campaña electoral, quien se dio cuenta de las posibilidades políticas de esa transparencia enunciada en la FOIA .El concepto radica en que un ciudadano informado de los asuntos públicos se compromete en el desarrollo político y social de su país y un Gobierno transparente, ofrece a los ciudadanos datos de su gestión para que estos puedan opinar y participar con conocimiento de causa y criterio (Naser \& Gastón, 2012).

En términos de institucionalidad las entidades más débiles fueron las alcaldías, los riesgos de corrupción administrativa se incrementan en la medida en que son más bajos el desarrollo institucional (que se cumplan normas y protocolos), la actuación de los controles y la difusión de la información de gestión. Las políticas anticorrupción no siempre están determinadas en estas entidades territoriales, no se perciben con claridad estrategias o lineamientos orientados a luchar contra la corrupción o a identificar los posibles riesgos. En este sentido (Católico \& Santos, 2018) citando (Saiz, Mantilla \& Cárdenas, 2011; Maldonado, 2011), indican que la corrupción se manifiesta en distintos ámbitos: el legislativo, correspondiente a las actuaciones que se llevan a cabo en las corporaciones públicas; el judicial, referido a las decisiones de los jueces; el administrativo, en relación con el abuso del cargo público, mediante el incumplimiento de normas jurídicas y el favorecimiento a quien otorga un beneficio; y el político, en el escenario del fraude electoral (pag. 45). Usualmente la descentralización fiscal y política es un elemento asociado a mayor riesgo de corrupción, situación que se percibe en los resultados.

En el análisis bibliométrico se estableció la relación entre la institucionalidad y el enfoque de gobernanza, y efectivamente puede determinarse una importante reciprocidad pues un sistema de normas y reglas establecido fortalece la institucionalidad, al respecto Martínez Nogueira (s.f.) en el documento, la institucionalidad y gobernanza: nuevas perspectivas para la gestión pública, menciona que "gobiernos con débiles capacidades, sociedades con carencias notorias de gobernabilidad e institucionalidad de baja calidad y con gobernanza problemática no contarán con normas básicas de funcionamiento que consoliden la integración social, la 
adecuada distribución de recursos sociales, den seguridad y previsibilidad a las transacciones económicas y canalicen los riesgos" (pág. 89).

Transparencia por Colombia (2019) indica que:

El proceso descentralizador requiere del fortalecimiento de las entidades territoriales, en especial, de aquellas de menor desarrollo institucional, de forma tal, que se le permita a las Alcaldías y Gobernaciones del país la conformación de plantas de personal suficientes y bien capacitadas para el cumplimiento adecuado de sus funciones y para la reducción de los riesgos de corrupción en la fusión pública" (pág. 19).

Al revisar los resultados del factor de control y sanción, se encontró debilidad en todas las entidades en los procesos de rendición de cuentas y atención al ciudadano, no hay una oportunidad en la respuesta a las solicitudes ciudadanas, ni unos adecuados canales de denuncia, la rendición de cuentas no siempre cuenta con diferentes espacios que potencialicen el diálogo y un seguimiento responsable del actual público, no se facilita la participación y control social, ni hay condiciones institucionales para la participación ciudadana mediante el diálogo es un aspecto a fortalecer en todos los niveles analizados. En este sentido La gobernabilidad se ve amenazada por la falta de credibilidad en el quehacer público, las reformas y políticas públicas son en ocasiones ineficaces, al no tener una suficiente participación de la ciudadanía respecto a lo que considere prioritario. Otro factor que hace que se pierda credibilidad en la actuación pública y en los mecanismos de control es un poder judicial débil que no ejerza control y penalización sobre los hechos de corrupción, esto puede evidenciarse en los resultados de la Fiscalía General de la Nación que es una entidad considerada con un nivel alto de corrupción, que justamente tiene su indicador más pobre en la variable de Control y Sanción.

Sin embargo, el masivo uso de internet y de redes sociales posibilita en mayor medida la opinión pública, los ciudadanos se expresan ahora más que antes y ejercen control, no por los mecanismos establecidos sino mediante la opinión, en este contexto los procesos de rendición de cuentas y de gobierno abierto facilitan la transparencia y el derecho a acceder a la información. Si bien es cierto, en Colombia se ha avanzado en torno a la normatividad, el gobierno en Línea y presentación de informes de las entidades públicas, se está lejos de desarrollar una verdadera gestión pública, que se oriente al ciudadano y su necesidades, aunque existe la información no se le saca provecho para direccionar los planes de gestión de las entidades, el valor de los datos es la información que se obtiene de ellos, la comprensión de la misma y su uso para el beneficio de los gobernados, una entidad pública visible, se mide por el grado en que divulga sus procesos de gestión administrativa, gestión presupuestal, tramites al ciudadano, facilitando el acceso a la información mediante páginas web.

Varias publicaciones han profundizado acerca de la relación entre el gobierno electrónico y la confianza ciudadana, sosteniendo que una mayor exposición en medios informáticos mejora la percepción ciudadana acerca del sector público. Grimmelikhuijsen y Feeney (2017), indican que existen tres dimensiones de gobierno abierto: la accesibilidad, la transparencia y la participación. La accesibilidad se refiere a la capacidad de las partes interesadas para utilizar la información o las opciones de participación en línea o fuera de ella, la transparencia se relaciona en como las organizaciones gubernamentales brindan información y la participación hace referencia a cómo los gobiernos permiten qué los ciudadanos interactúen con ellos de manera democrática. Se concluye que los tres factores se fomentan en culturas organizativas innovadoras, poco burocráticas y con capacidad tecnológica. 


\section{Conclusiones}

La transparencia es una obligación de las entidades públicas que tienen el deber de dar a conocer su actuar y gestión, se constituye en uno de los pilares de la democracia participativa se relaciona de manera directa con la ética. Aunque en el país se ha avanzado en términos de acceso a la información aún se requiere responder a demandas sociales y fortalecer la rendición de cuentas por diversos medios tecnológicos y no tecnológicos con el fin de favorecer la participación ciudadana y el flujo de la información entre el estado y sus gobernados.

Es importante resaltar que la percepción de transparencia y confianza en las entidades territoriales es muy baja tanto en América Latina como en el país, la única manera de superar esta situación es que los ciudadanos se empoderen e involucren en el quehacer público, es la participación ciudadana la que exige a los gobernantes a dirigir los bienes públicos con integridad, transparencia y con la obligación de rendir cuentas, no en vano las entidades más visibles son consideradas menos corruptas de acuerdo a los resultados estudiados.

Respecto al tema de visibilidad se aprecia en las entidades analizadas una mayor divulgación de la información financiera y presupuestal promovido por las acciones de gobierno electrónico que se han venido instaurando en el país, sin embargo, este acceso está limitado por el acceso a internet en las regiones y el interés de los ciudadanos en el quehacer de sus instituciones.

En términos de institucionalidad, los temas de planeación y contratación fueron mejor valorados, pero se destacó la desvalorización ante las políticas de anticorrupción, las cuales no se consideran efectivas, y en este punto se requiere fortalecer un marco político y estratégico que permita reformar sectores e instituciones hacia lo preventivo y lo sancionatorio cuando haya lugar. En procesos de control y sanción, el tema álgido en la mayoría de entidades consultas es el de rendición de cuentas, las autoridades gubernamentales están obligadas a rendir cuentas de manera oportuna y confiable y, la ciudadanía y la sociedad civil organizada deben participar activamente en estos procesos para exigir de sus gobernantes responsabilidad.

La percepción de transparencia lleva implícito el tema de la corrupción. En el país son contantes los escándalos que disminuyen la credibilidad en las instituciones redundando en una menor legitimidad institucional, generando desconfianza en los representantes de las instituciones y en el proceso democrático. La manera más eficaz de prevenir la corrupción es consolidar mecanismos de visibilidad, institucionalidad y control y sanción, para fortalecer la sensación de transparencia, aunque la percepción de transparencia es un fenómeno subjetivo y de difícil medición es importante, contar con cifras que permitan estudiar el fenómeno y ver su evolución para poder proponer lineamientos que fortalezcan la gobernanza.

La corrupción limita el crecimiento económico, afecta el desarrollo sostenido e impide brindar mejor calidad de vida para los ciudadanos, pues se dejan de satisfacer necesidades apremiantes de la sociedad al desviar los recursos a unos pocos. Hay un largo camino por recorrer en las instituciones públicas del país, pero una sociedad civil empoderada, participativa y atenta a procesos de rendición de cuentas, favorece el diseño de políticas públicas pertinentes que respondan a las necesidades de las regiones. 


\section{Referencias}

Arredondo Vélez, H. A. (2019). Los fundamentos morales y económicos de la corrupción. Panorama Económico, 14(28), 111. https://doi.org/10.29201/pe-ipn.v14i28.219

http://panoramaeconomico.mx/ojs/index.php/PE/article/view/219

Católico, D. y Santos, G. (2018) La corrupción y la transparencia de las administraciones públicas: un estudio empírico en territorios de paz en Colombia. Estudios de Derecho, 2018, vol. 75, no 166, p. 5176.https://dialnet.unirioja.es/servlet/articulo?codigo=6759867

Cerrillo I, Martínez, A. (2012). Transparencia administrativa y lucha contra la corrupción en la Administración local. http://repositorio.gobiernolocal.es/xmlui/handle/10873/1255

Corporación Transparencia por Colombia (2017) Índice de Transparencia Nacional 2015-2016 (Colección de Documentos Observatorio de Integridad N. 19)

http://indicedetransparencia.org.co/Portals/O/Documentos/Publicaciones\%202015- 2016/ITN\%2020152016.pdf

Cunill, N. (2003). Responsabilizacion por el Control social. Cuaderno de ciencias sociales No 128 Flasco . https://controlatugobierno.com/archivos/bibliografia/responsabilizacioncontrol.pdf

Cunill , N. (2010). Las políticas con enfoque de derechos y su incidencia en la institucionalidad pública. Revista del CLAD Reforma y Democracia, (46), 41-72. https://www.redalyc.org/pdf/3575/357533678002.pdf

Dassen, N., Vieyra, J. C., Molina, E., Ramírez Alujas, Á. V., Díaz Frers, L., Elena, S. y de Michele, R. (2012). Gobierno abierto y transparencia focalizada: tendencias y desafíos para América Latina y el Caribe. https://www.researchgate.net/profile/Juan_Vieyra/publication/332494886_Gobierno_Abierto_y_Transpa rencia_Focalizada_Tendencias_y_Desafios_para_America_Latina_y_el_Caribe/links/5cb80a1092851c8d22 f2ef87/Gobierno-Abierto-y-Transparencia-Focalizada-Tendencias-y-Desafios-para-America-Latina-y-elCaribe.pdf

Fox, J. (2007). The uncertain relationship between transparency and accountability. Development in Practice, 17(4), 663-671. https://www.researchgate.net/publication/46440861_The_Uncertain_Relationship_between_Transparen cy_and_Accountability

Grimmelikhuijsen, S. G., y Feeney, M. K. (2017). Developing and testing an integrative framework for open government adoption in local governments. Public Administration Review, 77(4), 579-590. https://onlinelibrary.wiley.com/doi/abs/10.1111/puar.12689

Transparencia por Colombia2017). Índice de Transparencia Municipal resultados 2015 - 2016 (https://transparenciacolombia.org.co/2017/03/27/indice-de-transparencia-municipal-2015-2016/

Kosack, S., y Fung, A. (2014). Does transparency improve governance?. Annual review of political science, 17, 65-87. https://www.annualreviews.org/doi/pdf/10.1146/annurev-polisci-032210-144356 
Martínez Nogueira, R.(s.f.). La institucionalidad y gobernanza:¿ nuevas perspectivas para la gestión pública?. Asociación de administradores gubernamentales, 55.

http://www.asociacionag.org.ar/pdfaportes/30/05\%20-\%20Roberto\%20Martinez\%20Nogueira\%20\%20La\%20institucionalidad\%20y\%20gobernanza.pdf

Martínez-Toro, G. M., Rico-Bautista, D., Romero-Riaño, E., Galeano-Barrera, C. J., Guerrero, C. D., \& ParraValencia, J. A. (2019). Análisis de la estructura intelectual y la evolución de la investigación en la interacción humano-computador: un análisis bibliométrico. Revista Ibérica de Sistemas e Tecnologias de Informação, (E17), 363-378. https://www.researchgate.net/profile/Dewar_Rico-

Bautista/publication/331178201_Analysis_of_the_intellectual_structure_and_evolution_of_research_in_ human-computer_interaction_A_bibliometric_analysis/links/5d09b2fa92851cfcc622bf80/Analysis-of-theintellectual-structure-and-evolution-of-research-in-human-computer-interaction-A-bibliometricanalysis.pdf

Naser, A. y Gastón Concha (2012). El desafío hacia el gobierno abierto en la hora de la igualdad; Santiago, CEPAL. p. 30. Documentos de Proyectos No.465 (LC/W.465) https://www.cepal.org/es/publicaciones/3969-desafio-gobierno-abierto-la-hora-la-igualdad

Naser, A. y Ramírez A. (2013). Plan de gobierno abierto: Una hoja de ruta para los gobiernos de la región, Santiago, CEPAL. 68 p. Serie Manuales No. 81, (LC/L.3802 - LC/IP/L.333) https://www.cepal.org/ilpes/noticias/paginas/3/54303/Plan_de_Gobierno_Abierto.pdf

Newman Pont, V. \& Ángel Arango, M. (2017) Sobre la corrupción en Colombia: marco conceptual, diagnóstico y propuestas de política. Bogotá: Fedesarrollo, 222 p. Cuadernos de Fedesarrollo No. 56 https://www.repository.fedesarrollo.org.co/handle/11445/3436

Ortiz Escobar, D. y Ordoñez Beltrán, S. (2018). Buena administración, transparencia y eficiencia: evidencia de los municipios de Colombia. Revista Digital de Derecho Administrativo. 21 (nov. 2018), 179-199. DOI:https://doi.org/10.18601/21452946.n21.09. https://revistas.uexternado.edu.co/index.php/Deradm/article/view/5704

Prats,J (1998). Administración pública y desarrollo en América Latina. Un enfoque neoinstitucionalista * Publicado en la Revista del CLAD Reforma y Democracia. No. 11 (Jun. 1998). Caracas. http://old.clad.org/portal/publicaciones-del-clad/revista-clad-reforma-democracia/articulos/011-junio1998/administracion-publica-y-desarrollo-en-america-latina.-un-enfoque-neoinstitucionalista-1

Philp, M. (2001). Access, accountability and authority: Corruption and the democratic process. Crime, Law and Social Change 36, 357-377. https://doi.org/10.1023/A:1012075027147 https://link.springer.com/article/10.1023/A:1012075027147

Pliscoff, C. \& Monje, P. (2003). Método comparado: un aporte a la investigación en gestión pública. In VIII Congreso Internacional del CLAD sobre la Reforma del Estado y de la Administración Pública. Panamá: Octubre.

https://www.researchgate.net/publication/322750334_Metodo_comparado_un_aporte_a_la_investigaci on_en_gestion_publica 
Rose-Ackerman, S. (2001) Corrupción y gobierno: causas, consecuencias y reforma. Madrid: Siglo XXI de España Editores.- 366 p., Recuperado de http://old.clad.org/otras-publicaciones/la-corrupcion-y-los-gobiernoscausas-consecuencias-y-reforma

Rothstein, B. (2013). Corruption and Social Trust: Why the Fish Rots from the Head Down. Social Research: An International Quarterly 80(4), 1009-1032. DOI: 10.17506 / ryipl.2016.17.1.3760 Recuperado de https://www.researchgate.net/publication/265812672_Corruption_and_Social_Trust_Why_the_Fish_Rots _From_the_Head_Down

Transparencia por Colombia (2019) Descentralización para fortalecer la lucha anticorrupción.https://transparenciacolombia.org.co/wp-content/uploads/recomendacionesdescentralizacion.pdf

\section{(cc) BY-NC}

